\title{
How to Schedule Elective Surgical Cases into Specific Operating Rooms to Maximize the Efficiency of Use of Operating Room Time
}

\author{
Franklin Dexter, MD, PhD*, and Rodney D. Traub, PhD + \\ *Division of Management Consulting, Department of Anesthesia, University of Iowa, Iowa City, Iowa; and tCollege of \\ Business Administration, North Dakota State University, Fargo, North Dakota
}

\begin{abstract}
We considered elective case scheduling at hospitals and surgical centers at which surgeons and patients choose the day of surgery, cases are not turned away, and anesthesia and nursing staffing are adjusted to maximize the efficiency of use of operating room (OR) time. We investigated scheduling a new case into an OR by using two patient-scheduling rules: Earliest Start Time or Latest Start Time. By using several scenarios, we showed that the use of Earliest Start Time is rational economically at such facilities. Specifically, it maximizes OR efficiency when a service has nearly filled its regularly scheduled hours of OR time. However, Latest Start Time will perform better at balancing workload among services' OR time. We then used historical case
\end{abstract}

duration data from two facilities in computer simulations to investigate the effect of errors in predicting case durations on the performance of these two heuristics. The achievable incremental reduction in overtime by having perfect information on case duration versus using historical case durations was only a few minutes per OR. The differences between Earliest Start Time and Latest Start Time were also only a few minutes per OR. We conclude that for facilities at which the goals are, in order of importance, safety, patient and surgeon access to OR time, and then efficiency, few restrictions need to be placed on patient scheduling to achieve an efficient use of OR time.

(Anesth Analg 2002;94:933-42)
$\mathbf{T}$ his article applies to the many hospitals and freestanding surgical centers at which surgeons and patients choose the day of surgery, elective cases are not turned away, and anesthesia and nursing staffing are adjusted to maximize the efficiency of use of operating room (OR) time (1-4). Such facilities need to balance OR workload among ORs each workday (5). Otherwise, hypothetically, a service could be allocated two ORs for the day but perform all of its cases in one OR. There could be both unused OR time in one OR and overtime in the other $(1,3)$.

A new elective case could be scheduled into the appropriate OR by use of one of two typical heuristics (i.e., sophisticated rules of thumb).

Earliest Start Time. The case is scheduled into the OR with the earliest start time.

Latest Start Time. The case is scheduled into the OR with the latest start time that is sufficiently early that the case will probably be completed during regularly

\footnotetext{
Supported by University of Iowa Health Care.

Accepted for publication December 19, 2001.

Address correspondence and reprint requests to Franklin Dexter, Division of Management Consulting, Department of Anesthesia, University of Iowa, Iowa City, IA 52242. Address e-mail to Franklin-Dexter@UIowa.edu.
}

scheduled OR time. Otherwise, the case is scheduled into the OR with the earliest start time.

Frequently, constraints (e.g., the surgeon following himself or herself in the same OR) will prevent a case from being scheduled by use of Earliest Start Time or Latest Start Time. Nevertheless, we studied Earliest Start Time and Latest Start Time because they are simple, rational, and (most important) two extreme policies. Earliest Start Time lets the surgeon and patient finish as early as possible and have a more predictable start time (6). Latest Start Time provides a better chance that if a new case were scheduled, it could be completed in regularly scheduled OR time (7).

In this article, we answer the following questions. First, what precisely are the economic rationales for these two heuristics? Do they maximize the efficiency of use of OR time, as is appropriate (1-3) for such surgical suites? Second, under what conditions is one preferable to the other for maximizing the efficiency of use of OR time? Third, under what conditions is one heuristic preferable to the other for balancing workload among different services' ORs on the same day? Fourth, how well do they perform in practice at maximizing the efficiency of use of OR time? What could 
be the incremental benefit of applying more sophisticated algorithms to this decision-making process? We start the Discussion by providing to OR managers specific guidelines for case scheduling to maximize OR efficiency.

\section{Background and Theoretical Analyses}

The purpose of this Background and Theoretical Analyses section is threefold. First, we review background information, such as precise definitions of OR management terms. Second, we use hypothetical scenarios to answer the first three of the four questions in the preceding paragraph. Third, we address the theoretical construct for the statistical analysis of the Methods and Results.

\section{Efficiency of Use of OR Time}

Efficiency is defined in terms of underutilized and overutilized hours of OR time $(1,3)$. For example, anesthesia and nursing staff members' regularly scheduled OR hours are 7:00 AM to 3:00 PM. The last case of the day in an OR ends at 1:00 PM. There are two underutilized hours of OR time $(1,3)$. If, instead, the last case of the day ends at 5:30 PM, then there are 2.5 overutilized OR hours.

The cost of overutilized OR time includes both the direct costs of overtime and the indirect costs of possible employee dissatisfaction, resignation, and recruitment $(2,4)$.

The efficiency of use of OR time is maximized by minimizing the sum of two products: underutilized OR hours multiplied by the cost per hour of underutilized OR time and overutilized OR hours multiplied by the cost per hour of overutilized OR time $(1,3)$.

This article's analyses are on a per-service basis. Typically, allocating OR time to services instead of individual surgeons results in higher OR efficiency (2).

\section{Interpreting Balancing OR Workload Among Different Surgical Services' ORs in Terms of the Efficiency of Use of OR Time}

We considered facilities at which the surgeons and patients choose the day of surgery, cases are not turned away, and OR efficiency is maximized. Because the surgeon and patient choose the day of surgery, if one service schedules cases into another service's OR time, that should not affect either service's ability to schedule additional cases for that workday.

Scenario $A$. The regularly scheduled workday is $8 \mathrm{~h}$. Service 1 has filled its OR 1 . Service 2 has scheduled a 2-h case in its OR 2. Service 1 has a new 3-h case. Earliest Start Time schedules the case into Service 2's OR 2. Subsequently, Service 2 has a new 5-h case. Earliest Start Time schedules the case into OR 2. OR 2 has $10 \mathrm{~h}$ of cases. The result is that Service 2 works $2 \mathrm{~h}$ in overutilized OR time because of Service 1.

Scenario B. The scenario is the same as A except that Service 2 schedules both of the new cases. The result is that Service 2 works $2 \mathrm{~h}$ in overutilized OR time because of Service 2 .

Scenario C. The scenario is the same as A except that the OR manager planned $10 \mathrm{~h}$ of staffing for OR 2 . The result is zero overutilized hours of OR time.

The indirect cost per hour of overutilized OR time may seem higher in Scenario A than B, because one service booked a case in another service's OR time. Scenario $C$ shows that this argument is flawed. The efficient use of OR time is a staffing- and patientscheduling issue, not one of organizational dynamics, politics, and so on.

Scenarios A-C show what to do if the objective is to maximize OR efficiency and a surgical service's OR time is full. Then, its cases should be scheduled into another service's OR time instead of in overutilized OR time. Obviously, constraints may make this impossible (e.g., the surgeon's being in two locations simultaneously).

\section{Economic Rationale for the Heuristics: One New Case and Deterministic Case Durations}

Deterministic case durations are known precisely. For example, if the scheduled duration of a case is $3.5 \mathrm{~h}$, then the case will last precisely $3.5 \mathrm{~h}$.

From the definitions of Earliest Start Time and Latest Start Time (see Introduction), these heuristics schedule a new case differently only when there are no resulting overutilized OR hours. Both heuristics achieve the same number of hours of underutilized OR time, equaling the difference between regularly scheduled hours of OR time and the total hours of cases. Consequently, when there is one new case to be scheduled and case durations are deterministic, Earliest Start Time and Latest Start Time achieve the same maximal OR efficiency.

By definition, Earliest Start Time and Latest Start Time schedule the case into the same earliest starting OR when overutilized OR time is inevitable. We prove in Appendix 1 that under this circumstance, they both maximize OR efficiency.

Together, these results show that when there is one new case and there are deterministic case durations, Earliest Start Time and Latest Start Time both maximize OR efficiency. Thus, we have shown that, to maximize OR efficiency, one new case should not be scheduled to be completed in overutilized OR time if it can start earlier in another OR. 


\section{Cost Per Hour of Underutilized OR Time a Few Days Before the Day of Surgery}

OR block time and staffing can be adjusted every several months to maximize expected OR efficiency $(1-3,8)$. On this long-term basis, the cost per hour of underutilized OR time equals the cost of staffing that hour (1-3).

A few days before the day of surgery and on the day of surgery, the full-time staff have been scheduled. Thus, the direct incremental cost per hour of underutilized OR time is zero. Because surgeons choose the day of surgery (1-3), cases are not queued (7) for empty OR time. Underutilized OR time does not represent lost revenue for the surgical suite. Thus, the opportunity cost of underutilized OR time also is zero. Consequently, a few days before surgery, maximizing the efficiency of use of OR time is synonymous with minimizing overutilized OR time.

For example, on the day of surgery when deciding whether to move the last case of the day from one late-running OR to another empty one, the reported objective (9) of minimizing overutilized OR time is the same as maximizing OR efficiency.

\section{Relative Performance of Earliest Start Time and Latest Start Time: One New Case}

Earliest Start Time balances underutilized OR time equally among ORs. Latest Start Time does the opposite, filling some ORs before starting to use others. Each additional scheduled case in an OR increases the chance of overutilized OR time. With use of the best methods known, to date, to predict case durations by using historical data, the mean \pm SE of absolute errors in the times to complete series of cases in an OR are substantial (1.63 $\pm 0.03 \mathrm{~h})(10)$. Consequently, for one new case, Earliest Start Time performs better on average than Latest Start Time at minimizing overutilized OR hours (i.e., from Section 4, at maximizing OR efficiency). This is a different result from that in Section 3 , because in this section we are not assuming that case durations are deterministic.

Earliest Start Time also performs better on average than Latest Start Time at letting surgeons finish their work sooner and reducing patients' total waiting time on the day of surgery.

This article applies to surgical suites that do not routinely schedule delays between different surgeons' cases in the same OR on the same day, because that would reduce OR efficiency (11). Consequently, Latest Start Time increases the risk that preceding cases in an OR will finish late and prevent the on-time start of the new, last case $(6,10)$. Thus, Earliest Start Time performs better on average than Latest Start Time at reducing patients' and surgeons' waiting time caused by late starts on the day of surgery.

\section{Characteristics of the Filling of OR Time by Individual Surgical Services}

In Methods and Results, we use historical data to compare the average difference in overutilized OR time resulting from using Earliest Start Time with estimated case durations versus with perfect knowledge of case durations. We simulate the scheduling of one new case into ORs. In this section, we consider why considering zero cases or one new case is appropriate.

When one or both simulated ORs are nearly empty, neither Earliest Start Time nor scheduling by using perfect knowledge will produce overutilized OR time. Earliest Start Time with historical case duration data will produce significantly more overutilized OR time than scheduling with perfect knowledge only when both ORs are nearly full. Once a service's regularly scheduled OR time is nearly full, how many new cases are scheduled on average by a service? The relevant aspect of OR patient scheduling (for readers from nonmedical specialties) is that average durations for all cases performed in a surgical suite are relatively long compared with regularly scheduled OR hours. Among surgical suites in the United States, there is an average of 2.0 cases per OR each workday (12).

As OR time fills, there is a declining probability that the service will schedule another case, because the original allocation was made to maximize OR efficiency. Because underutilized OR time is less expensive than overutilized OR time, the probability is not only more than $50 \%$ that each OR will have underutilized OR time, but also that each service's average hours of underutilized OR time will exceed its average hours of overutilized OR time $(1,3)$. Suppose that a service has $14 \mathrm{~h}$ of cases scheduled into its $16 \mathrm{~h}$ of OR time. The probability that the service will schedule another case is small, because otherwise the service would have been allocated more than $16 \mathrm{~h}$ of OR time.

There is also a behavioral component to patient scheduling. When a service's regularly scheduled OR time on a potential day of surgery is nearly full, the surgeon and patient can schedule the new case to be completed in overutilized OR time or schedule the case to start earlier on another day of surgery. We reiterate that our analysis applies only to surgical suites at which the surgeon and patient can choose any workday for the case. As a service's OR time fills on one day, there is a declining probability that its surgeons and patients will schedule another case on that day, because (as considered in Section 5) from the surgeons' and patients' perspectives, it is advantageous to perform cases early in the day. 


\section{Rationale for Evaluating Performance of Earliest Start Time When There Is One New Case}

Section 5 showed that Earliest Start Time will perform better on average than Latest Start Time at maximizing OR efficiency and other criteria. Nonetheless, Earliest Start Time may still perform poorly in practice when case durations are estimated from averages of historical case durations. More sophisticated algorithms consider not only estimated start times, but the variability of the estimated start times $(6,11,13,14)$.

Scenario D. A surgeon wants to schedule a laparoscopic cholecystectomy. In OR 1, a different surgeon has scheduled two laparoscopic cholecystectomies. In OR 2, a radical resection of tumor of the shaft of the humerus has been scheduled. Mean \pm SD values of historical case durations for these procedures are $2.7 \pm$ $0.7 \mathrm{~h}(n=552)$ and $4.7 \pm 1.0 \mathrm{~h}(n=2)$, respectively. Turnover times at the hospital are $0.5 \pm 0.2 \mathrm{~h}(n=$ $12,163)$. The regularly scheduled OR hours are 7:00 AM to 3:00 PM. The estimated start times for the new cholecystectomy would be 1:24 PM in OR 1 (7:00 AM + $2 \times 2.7 \mathrm{~h}+2 \times 0.5 \mathrm{~h})$ and 12:12 $\mathrm{PM}$ in OR 2 (7:00 $\mathrm{AM}$ $+4.7 \mathrm{~h}+0.5 \mathrm{~h})$.

Earliest Start Time schedules the new case to follow the tumor resection in OR 2, because it considers the expected start time of the new case. Earliest Start Time does not "know" that orthopedic tumor resections have unpredictable durations and that only two historical case durations were used to estimate the start time. Thus, Earliest Start Time does not "know" that the estimated start time of 12:12 PM is less reliable than the 1:24 PM start time. The average overutilized OR time is, in fact, less if the new case is scheduled into OR $1(6,11,13)$.

Case durations consistently follow log-normal distributions $(6,11,13,15)$. This a priori knowledge can be used by more sophisticated methods of decisionmaking. These include Monte-Carlo simulation $(6,11)$ and linear programming (14) applied to the shortest $(6)$ and longest $(11,13)$ times to complete the preceding cases in the ORs.

\section{Comparison of Earliest Start Time and Latest Start Time When There Are Two or More New Cases and Deterministic Case Durations}

The arguments in Sections 3 and 5 about the advantages of Earliest Start Time over Latest Start Time do not apply when there are two or more new cases.

Scenario E. Services 1 and 2 each have been allocated one 8-h OR. Service 1 has previously scheduled $5 \mathrm{~h}$ of cases into its OR 1 . Service 2 has $4 \mathrm{~h}$ of cases in its OR 2. A surgeon from Service 1 wants to schedule a 3-h case. Earliest Start Time schedules the case into OR 2. There are then $5 \mathrm{~h}$ of cases in OR 1 and $7 \mathrm{~h}$ of cases in OR 2. Later, a surgeon from Service 2 wants to schedule a 3-h case. Earliest Start Time schedules the case into OR 1 . The result is zero overutilized hours.

Scenario F. This scenario is identical to E, except that the cases are scheduled by using Latest Start Time. The result is zero overutilized hours.

When the second new case is shorter than or of the same duration as the first new case, Earliest Start Time and Latest Start Time perform equally well.

Scenario G. Services 1 and 2 each have one 8-h OR. Service 1 schedules its cases 3 wk ahead of time. Service 2 schedules its cases 3 days ahead of time. Service 1 has scheduled $6 \mathrm{~h}$ of cases into its OR 1. Service 2 has scheduled no cases into its OR 2 . Service 1 has a new 2-h case. Earliest Start Time schedules it into OR 2. Two weeks later, Service 2 has a 4-h case and a 3-h case to schedule. Earliest Start Time schedules them into OR 2. The result is one overutilized hour of OR time.

Scenario $H$. The scenario is identical to G, except that Latest Start Time is used. The result is zero overutilized hours of OR time.

When the new case is longer than the first new case, Latest Start Time provides for the more efficient use of OR time than does Earliest Start Time.

In general, if the new cases add to a total duration less than or equal to that of the first new case, Earliest Start Time will perform as well or better than Latest Start Time at minimizing overutilized OR time and maximizing OR efficiency. If the new cases add to a total duration exceeding that of the first new case, Latest Start Time will perform as well as or better than Earliest Start Time.

These results apply to balancing workload among different surgical services' ORs. Because the surgeons and patients choose the day of surgery, the average number of days that patients wait for surgery once they have chosen to have surgery may vary among services (16). Although in Scenarios G and H, Service 1 is unlikely to have another case to schedule (Section 6), Service 2 did.

Thus, we have shown that, when balancing workload among services, a case should be scheduled into its own service's OR time if the case can be done in regularly scheduled OR time.

\section{Methods}

The purpose of the Methods is to use historical case duration data from two facilities in computer simulations to investigate the effect of errors in predicting case durations on the performance of case-scheduling heuristics.

We used 3 yr of historical case duration data from two facilities: a hospital surgical suite and an ambulatory surgery center. The cases were sorted by their 
scheduled Current Procedural Terminology code(s). Then, for each of these scheduled procedure(s), the cases were sorted by surgeon. Then, for each scheduled procedure(s) and surgeon, the cases were sorted by type of anesthesia (not monitored anesthesia care, monitored anesthesia care, or surgeon-administered local anesthesia only) (17). Finally, for each combination of scheduled procedure(s), surgeon, and type of anesthesia, the cases were sorted by date of the case. We divided the data into two portions: the first $2.75 \mathrm{yr}$ of cases to predict case durations and the most recent 3 mo of cases to test the heuristics.

Case duration was defined as the time from when the patient entered an OR until he or she left the OR. For each case in the most recent $3 \mathrm{mo}$, we calculated the mean of the durations of previous (from $2.75 \mathrm{yr}$ ) cases of the same scheduled procedure, surgeon, and anesthetic $(10,17)$. If two such previous cases were not available, we used the scheduled procedure and surgeon (10). If two such previous cases were not available, we used the scheduled procedure (18). If two such previous cases were not available, the case was not used in the next step of the analysis.

The steps in Appendix 2 were performed separately six times (two facilities $\times$ three heuristics: Earliest Start Time, Latest Start Time, and scheduling the case at random). The end point of each analysis was the mean of the difference in overutilized OR time between using the heuristic with estimated case durations versus using perfect knowledge of case duration to always schedule the case into the correct OR. This perfect knowledge was available to us because we knew, in retrospect, how long each case took.

We describe, in the fourth and fifth steps of Appendix 2, how we calculated overutilized OR hours. To do so, we needed to specify a duration for the regularly scheduled workday. For the ambulatory surgery center, we planned a 7-h workday. For the hospital surgical suite, we repeated the six analyses with 8-, 9-, and 10-h workdays. We report the percentage of simulated ORs that had overutilized hours.

\section{Results}

For the ambulatory surgery center, by planning a $7-\mathrm{h}$ workday, $18.2 \% \pm 0.3 \%$ of the ORs would have overutilized OR hours. Earliest Start Time had an excess of $1.0 \pm 0.1 \mathrm{~min}$ of overutilized time per OR compared with perfect knowledge of case durations. This result can be compared with the mean case duration of $98 \pm$ $1 \mathrm{~min}$ and absolute error in case duration prediction of $26 \pm 1 \mathrm{~min}$. Latest Start Time produced $0.4 \pm 0.1$ more minutes of overutilized time per OR than did Earliest Start Time.

For the hospital surgical suite, by planning 8-, 9-, or 10 -h workdays, $44.6 \% \pm 0.4 \%, 35.1 \% \pm 0.3 \%$, or $26.4 \%$ $\pm 0.3 \%$ of the ORs would have overutilized OR time, respectively. Earliest Start Time had an excess of $6.7 \pm$ $0.4 \mathrm{~min}, 5.4 \pm 0.3 \mathrm{~min}$, or $4.2 \pm 0.3 \mathrm{~min}$ of overutilized time per OR, respectively, versus having perfect knowledge of case duration. These results can be compared with the mean case duration of $218 \pm 1 \mathrm{~min}$ and absolute error in case duration prediction of $51 \pm$ $1 \mathrm{~min}$. Latest Start Time produced $1.9 \pm 0.4,2.6 \pm 0.4$, and $3.6 \pm 0.4$ more minutes of overutilized OR time per OR, respectively, than did Earliest Start Time.

An OR manager could leave patient scheduling to chance (i.e., the OR into which the case was scheduled was chosen at random). For the ambulatory surgery center, with random scheduling, there would be an excess of $13.7 \pm 0.4 \mathrm{~min}$ of overutilized OR time per OR as compared with having perfect knowledge of case duration. For the hospital surgical suite, there would be $52.4 \pm 1.1,41.1 \pm 1.0$, and $30.1 \pm 0.8$ extra minutes of overutilized OR time per OR, respectively, versus having perfect knowledge. These four differences significantly exceeded the corresponding differences between Earliest Start Time and Latest Start Time. On the basis of the definitions of Earliest Start Time and Latest Start Time, this can be accounted for only by cases being scheduled into ORs such that estimated completion times were in overutilized OR time even though the other OR provided for an earlier start time.

\section{Discussion}

\section{Implications: How to Schedule Elective Surgical Cases to Maximize OR Efficiency}

When a surgical case is scheduled, Rule 1 needs to be followed to maximize OR efficiency.

Rule 1 (from Section 2). If the service has already filled its regularly scheduled OR time, the new case should be scheduled into another service's regularly scheduled OR time instead of in overutilized OR time.

For example, Service 1 has filled its allocated OR time. Service 2 has not. Service 1 has a new case. It should be scheduled into Service 2's OR time. Obviously, sometimes this will not be possible because of constraints (e.g., if Service 1 is cardiac surgery and Service 2 is ophthalmology with a suspended microscope in its OR). Rule 1 shows that having a predetermined "release time" for a service's allocated OR time will result in reduced OR efficiency.

Provided that Rule \#1 does not apply, the next step is to apply Rules 2 and 3.

Rule 2 (from Section 8). A service should not schedule a case into another service's OR time if the case can be completed within its own regularly scheduled OR time. For example, Service 1 has four hours of allocated but unscheduled OR time. Service 1 should 
schedule its new two-hour case into its own OR time. We showed that this rule can be justified on the basis of OR efficiency, versus invoking some other (emotional) criteria.

Rule 3 (from Section 3 and Results). A case should not be scheduled to be completed in overutilized OR time if it can start earlier in another of the service's ORs. For example, Service 2 has filled its OR 1, has no cases scheduled into its OR 2, and has a new case to be scheduled. The new case should be scheduled into OR 2, not OR 1.

Patients and surgeons may naturally schedule new cases into their service's OR providing the Earliest Start Time (Section 6). This, conveniently, maximizes OR efficiency (Section 5). Nevertheless, provided the case is expected to be finished within the service's regularly scheduled OR time, the Results show that the choice of the OR into which the case is scheduled has a financially unimportant effect on OR efficiency. This is because a few minutes of additional overutilized OR time per OR are negligible. In addition, the Results show that neither Earliest Start Time nor Latest Start Time is preferable to the other in terms of OR efficiency. Thus, Rules 2 and 3 specify that the OR manager can achieve efficient OR scheduling while leaving case-scheduling decisions to the convenience of the surgeons and patients, provided that each case is scheduled into its service's allocated OR time.

\section{Communicating OR Strategic Goals to Stakeholders}

Maximizing the efficiency of use of OR time is important to OR managers because it is tied closely to the profitability of the facility and anesthesia group (2).

Maximizing OR efficiency is also an important basis for OR management decision-making. Specifically, safety needs to be the top priority for decisionmaking. Otherwise, for example, unsterilized instruments would be used on a patient because the surgeon wanted to do the case now. For the surgical suites considered in this article, caring for all of the surgeons' patients every day has to be the second priority. Otherwise, aiming to maximize the efficiency of use of OR time would result in there being fixed hours of OR time. Maximizing OR efficiency is left as the third goal.

These three goals (safety, access, and then OR efficiency) are sufficient for most OR operational decisions. Strum et al. $(1,3)$ previously showed that OR allocations can be made to maximize OR efficiency. We previously showed that decisions on second-shift staffing (4), moving cases on the day of surgery (9), and assigning second-shift staff on the day of surgery (19) can be made to maximize OR efficiency. In this study, we showed that the allocation of separate OR time to different services can serve to maximize OR efficiency. We also showed that cases can be scheduled to maximize OR efficiency. Safety, access, and efficiency, in that order of importance, can be a simple message from the OR manager regarding a surgical suite's goals.

\section{Development and Implementation of More Sophisticated Case Scheduling Algorithms}

The best that more sophisticated case scheduling algorithms could achieve would be perfection. Nonetheless, the incremental amount of overutilized OR time achieved by using Earliest Start Time was small versus having perfect retrospective knowledge of case duration. Consequently, more sophisticated algorithms can achieve little relative to Earliest Start Time. Thus, we think that the incremental benefits of the more sophisticated algorithms are too small to warrant implementation. In addition, typically these algorithms (MonteCarlo simulation and linear programming) $(6,11,14)$ are nonintuitive and computationally intensive. Other factors are more important causes of OR inefficiency.

\section{Application to Surgical Suites with Fixed Hours of OR Time}

The conclusions of this article are different from those for facilities with limited hours of OR time (7). For such surgical suites, Latest Start Time performs substantively better than Earliest Start Time at maximizing the efficiency of use of OR time (7). The results are different from those we found in this article because the objective was different. When all patients receive care, the short-term objective in maximizing OR efficiency is to reduce overutilized OR time (Section 4). In contrast, with fixed hours of OR time, the short-term objective in maximizing OR efficiency is to reduce underutilized OR time (7). If the facility, anesthesia group, or both are fee-for-service or if they simply want to provide as much patient care as possible within a limited budget of OR time, then it is important that the fixed hours of OR time be as full as possible. OR managers at hospitals with fixed hours need to ensure that the simple and intuitive Earliest Start Time heuristic not be used by well intentioned schedulers and surgeons (7).

\section{Application to Surgical Services with Different Hours of Staffing Planned for Each Room}

We considered for convenience, but without loss of generality, that ORs have the same planned hours of staffing. OR nurses or anesthesia groups may plan different staffing for different ORs. For example, a surgical service with three ORs each Monday may have two planned for 8 hours and one for 13 hours. This has no effect on our results. OR managers may find it convenient under these circumstances to think 
of the Earliest Start Time rule as the most-timeremaining rule and the Latest Start Time rule as the least-time-remaining rule.

\section{Deciding into Which Surgical Service's OR Time a Case Should Be Scheduled from Another Service That Has Filled Its Allocated OR Time}

If one service has filled its allocated OR time and yet has another case to schedule, the findings of Section 2 show that the case should be scheduled into another service's OR time, provided that constraints do not make this impossible. Sections 4 and 8 provide some insight into how to decide into which OR to schedule the case. On a short-term basis, the objective of maximizing the efficiency of use of OR time is to minimize overutilized hours of OR time. Thus, when deciding into which OR to schedule the case, the OR manager should estimate the likelihood that each of the other services will subsequently schedule a sufficient number of hours of cases to then have overutilized OR time. Many other criteria may be considered in this decision by the OR manager (e.g., who is least likely to complain publicly). However, our recommended criterion maximizes OR efficiency.

\section{Limitations}

The focus of this article was on using case scheduling to maximize OR efficiency. Nonetheless, the most important way to maximize OR efficiency is to allocate OR time and plan staffing optimally $(2,20)$. The economic importance of OR management decisions made a few days before the day of surgery (e.g., those in this article) or on the day of surgery $(9,19)$ are small compared with those made months before the day of surgery $(1,3,4)$.

We estimated case duration by using the sample mean of the durations of cases of the same scheduled procedure(s) $(10,17,18)$. When possible, we limited consideration to the surgeon performing the case and the anesthetic used (17). Experimental studies have shown that permitting the surgeon to adjust the estimate up or down by a reasonable percentage (e.g., $10 \%$ ) to reflect case complexity can reduce error in predicting case duration (21). We were unable to examine this because of our study design. However, it seems logical to use this approach. Providing surgeons with this opportunity may be disadvantageous at facilities at which surgeons have a financial incentive to underestimate case durations. However, our study applies to facilities at which the surgeon and patient choose the day of surgery and cases are not turned away. Thus, there is no incentive to being inaccurate.

There are three stages to investigating management decision-making. The first is to characterize the problem mathematically. The second is to determine the optimal solution to solve that problem or to develop and test a suitable heuristic for the problem. The third is to evaluate how much better the optimal method or heuristic performs than what schedulers are doing in practice. We do not have the data to perform this third step. We also doubt that our three recommendations (see Implications, previously) are better than what schedulers are doing in practice. We think that our results are typical practice. This was not by design but just happened to be our results. The implications for OR managers may be that focusing organizational decision-making on maximizing OR efficiency may be easy to accomplish.

\section{Conclusions}

We showed how cases should be scheduled to maximize the efficiency of use of OR time at facilities for which surgeons and patients have access to OR time every workday. OR managers can generally leave case-scheduling decisions to the convenience of the surgeons and patients. However, to maximize OR efficiency, three scheduling ground rules must be followed.

\section{Appendix 1}

In this appendix, we show that if the time to complete a series of consecutive cases in one OR is longer than the time to complete a series of consecutive cases in another OR, then scheduling a new case into the OR providing the earliest start time will maximize the efficiency of use of OR time. This is true even if the cost per hour of underutilized OR time is not zero.

We use $T_{\text {new }}$ to represent the duration of the new case, including its preceding turnover time. The time to complete the series of cases currently scheduled into OR 1 is designated $T_{1}$. The corresponding time for OR 2 is designated $T_{2}$. The cost per hour of overutilized time is represented by $C_{\mathrm{o}}$. The cost per hour of underutilized time is represented by $C_{\mathrm{u}}$. The duration of the regularly scheduled workday is $T_{\text {shift }}$. The costs of scheduling the case into OR 1 or 2 are represented by $C_{1}$ and $C_{2}$, respectively.

The efficiency of use of OR time is maximized when costs are minimized. The costs to be minimized are the weighted sums of the underutilized and overutilized hours of OR time.

If the case was scheduled into OR 1 , then

$$
\begin{aligned}
& C_{1}=C_{\mathrm{u}} \max \left[0, T_{\text {shift }}-\left(T_{1}+T_{\text {new }}\right)\right] \\
& \quad+C_{\mathrm{o}} \max \left(0, T_{1}+T_{\text {new }}-T_{\text {shift }}\right) \\
& +C_{\mathrm{u}} \max \left(0, T_{\text {shift }}-T_{2}\right)+C_{\mathrm{o}} \max \left(0, T_{2}-T_{\text {shift }}\right) .
\end{aligned}
$$


If the case was scheduled into OR 2, then

$$
\begin{aligned}
& C_{2}=C_{\mathrm{u}} \max \left[0, T_{\text {shift }}-\left(T_{2}+T_{\text {new }}\right)\right] \\
& \quad+C_{\mathrm{o}} \max \left(0, T_{2}+T_{\text {new }}-T_{\text {shift }}\right) \\
& +C_{\mathrm{u}} \max \left(0, T_{\text {shift }}-T_{1}\right)+C_{\mathrm{o}} \max \left(0, T_{1}-T_{\text {shift }}\right) .
\end{aligned}
$$

To demonstrate that the use of OR time is maximized, we need to show that if $T_{1} \geq T_{2}$, then $C_{1} \geq C_{2}$. We consider three general conditions, some of which have subconditions. The three general conditions are $T_{1} \geq T_{2} \geq T_{\text {shift, }}, T_{\text {shift }} \geq T_{1} \geq T_{2}$, and $T_{1} \geq T_{\text {shift }} \geq T_{2}$.

\section{Condition 1: $\mathrm{T}_{1} \geq \mathrm{T}_{2} \geq \mathrm{T}_{\text {shift }}$}

Subject to this condition, Equation 1 can be written as

$$
C_{1}=C_{\mathrm{o}}\left(T_{1}+T_{\text {new }}-T_{\text {shift }}\right)+C_{\mathrm{o}}\left(T_{2}-T_{\text {shift }}\right),
$$

and Equation 2 can be written as

$$
C_{2}=C_{\mathrm{o}}\left(T_{2}+T_{\text {new }}-T_{\text {shift }}\right)+C_{\mathrm{o}}\left(T_{1}-T_{\text {shift }}\right) .
$$

Because Equations 3 and 4 are the same, when $T_{1} \geq T_{2}$ $\geq T_{\text {shift }}, C_{1}=C_{2}$.

Condition 2: $\mathrm{T}_{\text {shift }} \geq \mathrm{T}_{1} \geq \mathrm{T}_{2}$

Subject to this condition, Equation 1 can be written as

$$
\begin{aligned}
& C_{1}=C_{\mathrm{u}} \max {\left[0, T_{\text {shift }}-\left(T_{1}+T_{\text {new }}\right)\right] } \\
&+C_{\mathrm{o}} \max \left(0, T_{1}+T_{\text {new }}-T_{\text {shift }}\right) \\
& \quad+C_{\mathrm{u}}\left(T_{\text {shift }}-T_{2}\right),
\end{aligned}
$$

and Equation 2 can be written as

$$
\begin{aligned}
C_{2}=C_{\mathrm{u}} \max & {\left[0, T_{\text {shift }}-\left(T_{2}+T_{\text {new }}\right)\right] } \\
& +C_{\mathrm{o}} \max \left(0, T_{2}+T_{\text {new }}-T_{\text {shift }}\right) \\
& \quad+C_{\mathrm{u}}\left(T_{\text {shift }}-T_{1}\right) .
\end{aligned}
$$

To demonstrate that $C_{1} \geq C_{2}$, we separate the costs of overutilized OR time from the costs of underutilized OR time. We will show that the costs of overutilized OR time associated with $C_{1}$ are at least as large as the costs of overutilized OR time associated with $C_{2}$. We will then also show that the costs of underutilized OR time associated with $C_{1}$ are at least as large as the costs of underutilized OR time associated with $C_{2}$. Because the costs of overutilized OR time and underutilized OR time are being considered separately, it is sufficient to compare independently the overutilized and underutilized OR times in Equations 5 and 6.

Considering the costs of overutilized OR time, we combine the overutilized OR time components of Equations 5 and 6 into Equation 7. Because $T_{1} \geq T_{2}$,

$$
\begin{aligned}
C_{\mathrm{o}} \max \left(0, T_{1}+\right. & \left.T_{\text {new }}-T_{\text {shift }}\right) \\
& \geq C_{\mathrm{o}} \max \left(0, T_{2}+T_{\text {new }}-T_{\text {shift }}\right) .
\end{aligned}
$$

Consequently, the cost of overutilized OR time contributing to $C_{1}$ is at least equal to the cost of overutilized OR time contributing to $C_{2}$.

To consider the costs of underutilized OR time contributing to $C_{1}$ and $C_{2}$, we combine the underutilized OR time components of Equations 5 and 6 into Equation 8 .

$$
\begin{aligned}
C_{\mathrm{u}} \max \left[0, T_{\text {shift }}-\left(T_{1}+T_{\text {new }}\right)\right] & +C_{\mathrm{u}}\left(T_{\text {shift }}-T_{2}\right) \\
\geq C_{\mathrm{u}} \max \left[0, T_{\text {shift }}-\right. & \left.\left(T_{2}+T_{\text {new }}\right)\right] \\
& +C_{\mathrm{u}}\left(T_{\text {shift }}-T_{1}\right) .
\end{aligned}
$$

Although we have written Equation 8 with a " $\geq$ " symbol, we have not shown that this condition holds. To do so, we will consider three subconditions. For each we will show that Equation 8 is satisfied. That is, we will show that the cost of underutilized OR time in $C_{1}$ is at least equal to the cost of underutilized OR time in $C_{2}$. For convenience, we divide both sides of Equation 8 through by $C_{\mathrm{u}}$.

The first subcondition is $T_{\text {shift }} \geq T_{1}+T_{\text {new }} \geq T_{2}+$ $T_{\text {new }}$. Subject to this condition, Equation 8 can be written as

$$
\begin{aligned}
T_{\text {shift }}-\left(T_{1}+T_{\text {new }}\right) & +\left(T_{\text {shift }}-T_{2}\right) \\
\geq & T_{\text {shift }}-\left(T_{2}+T_{\text {new }}\right)+\left(T_{\text {shift }}-T_{1}\right) .
\end{aligned}
$$

Because by rearranging terms the two sides of the inequality are equal, Equation 8 is satisfied.

The second subcondition is $T_{1}+T_{\text {new }} \geq T_{2}+T_{\text {new }}$ $\geq T_{\text {shift }}$ Subject to this condition, Equation 8 can be written as

$$
\left(T_{\text {shift }}-T_{2}\right) \geq\left(T_{\text {shift }}-T_{1}\right) .
$$

Because Condition 2 specifies that $T_{1} \geq T_{2}$, the inequality holds. Equation 8 is satisfied.

The third subcondition is $T_{1}+T_{\text {new }} \geq T_{\text {shift }} \geq T_{2}+$ $T_{\text {new. }}$. Subject to this condition, Equation 8 can be written as

$$
\left(T_{\text {shift }}-T_{2}\right) \geq T_{\text {shift }}-\left(T_{2}+T_{\text {new }}\right)+\left(T_{\text {shift }}-T_{1}\right) .
$$

Subtracting $\left(T_{\text {shift }}-T_{2}\right)$ from both sides and adding $\left(T_{\text {new }}+T_{1}\right)$ to both sides,

$$
T_{1}+T_{\text {new }} \geq T_{\text {shift }} .
$$

Because the subcondition specifies that this condition is satisfied, Equation 8 is satisfied.

Thus, we have shown that subject to Condition 2 $\left(T_{\text {shift }} \geq T_{1} \geq T_{2}\right), C_{1} \geq C_{2}$. 
Condition 3: $\mathrm{T}_{1} \geq \mathrm{T}_{\text {shift }} \geq \mathrm{T}_{2}$

Subject to this condition, Equation 1 can be written as

$$
C_{1}=C_{\mathrm{o}}\left(T_{1}+T_{\text {new }}-T_{\text {shift }}\right)+C_{\mathrm{u}}\left(T_{\text {shift }}-T_{2}\right),
$$

and Equation 2 can be written as

$$
\begin{aligned}
C_{2}=C_{\mathrm{u}} \max \left[0, T_{\text {shift }}-\left(T_{2}+T_{\text {new }}\right)\right] & \\
+C_{\mathrm{o}} \max \left(0, T_{2}+\right. & \left.T_{\text {new }}-T_{\text {shift }}\right) \\
& +C_{\mathrm{o}}\left(T_{1}-T_{\text {shift }}\right) .
\end{aligned}
$$

The approach that we use is similar to that of Condition 2. We separate the overutilized OR time and the underutilized OR time and consider each separately.

The costs of underutilized OR time components of Equations 9 and 10 are

$$
C_{\mathrm{u}}\left(T_{\text {shift }}-T_{2}\right) \geq C_{\mathrm{u}} \max \left[0, T_{\text {shift }}-\left(T_{2}+T_{\text {new }}\right)\right] .
$$

Because $T_{\text {new }}>0$, the inequality is satisfied.

To consider the costs of overutilized OR time contributing to $C_{1}$ and $C_{2}$, we combine the overutilized OR time components of Equations 9 and 10 into Equation 12:

$$
\begin{array}{r}
C_{\mathrm{o}}\left(T_{1}+T_{\text {new }}-T_{\text {shift }}\right) \geq C_{\mathrm{o}} \max \left(0, T_{2}+T_{\text {new }}-T_{\text {shift }}\right) \\
+C_{\mathrm{o}}\left(T_{1}-T_{\text {shift }}\right) .
\end{array}
$$

Although we have written Equation 12 with a " $\geq$ " symbol, we have not shown that this condition holds. To do so, we will consider two subconditions. For each, we will show that Equation 12 is satisfied. That is, we will show that the cost of overutilized OR time in $C_{1}$ is at least as great as the cost of overutilized OR time in $C_{2}$. For convenience, we divide both sides of Equation 12 through by $C_{0}$.

The first subcondition is $T_{1}+T_{\text {new }} \geq T_{\text {shift }} \geq T_{2}+$ $T_{\text {new }}$. Subject to this condition, Equation 12 can be written as

$$
\left(T_{1}+T_{\text {new }}-T_{\text {shift }}\right) \geq\left(T_{1}-T_{\text {shift }}\right) .
$$

Because $T_{\text {new }}>0$, the subcondition is satisfied. Equation 12 is satisfied.

The second subcondition is $T_{1}+T_{\text {new }} \geq T_{2}+T_{\text {new }}$ $\geq T_{\text {shift }}$ Subject to this condition, Equation 12 can be written as

$$
\begin{aligned}
&\left(T_{1}+T_{\text {new }}-T_{\text {shift }}\right) \geq\left(T_{2}+T_{\text {new }}-T_{\text {shift }}\right) \\
&+\left(T_{1}-T_{\text {shift }}\right) .
\end{aligned}
$$

Subtracting $\left(T_{1}+T_{\text {new }}-T_{\text {shift }}\right)$ from both sides of the equation,

$$
0 \geq T_{2}-T_{\text {shift }} .
$$

Because Condition 3 specifies that this inequality is satisfied, Equation 12 is satisfied.

Thus, we have shown that subject to Condition $3\left(T_{1}\right.$ $\left.\geq T_{\text {shift }} \geq T_{2}\right), C_{1} \geq C_{2}$.

\section{Appendix 2}

This appendix describes how we calculated mean differences in overutilized OR time between using specified heuristics with estimated case durations versus using perfect knowledge of case duration to always schedule the case into the correct OR. These nine steps were performed six times, for the two facilities and for the three heuristics referred to in Step 3. All analyses were performed with Excel Visual Basic 6.0 (Microsoft, Redmond, WA).

1. Two series of consecutive elective cases in the same OR on the same day with the turnover time not exceeding $1 \mathrm{~h}$ from the most recent 3-mo period were selected at random and with replacement from all such series of cases during the most recent 3-mo period. This was done with Visual Basic's pseudouniformly distributed random number generator. The expected durations of these two series were calculated from the means of the cases' procedures' historical durations from the $2 \mathrm{yr}$ and 9 mo of data $(n=44,024$ cases). These random selections of two consecutive series of cases were from a population of 465 series of cases for the ambulatory surgery center and 1149 for the hospital surgical suite.

2. A case was selected at random from the most recent 3-mo period of cases. A turnover time was selected at random and added to the case. The expected durations were also obtained from the 2 yr and 9 mo of data. This random selection of a case was from a population of 1051 cases for the ambulatory surgery center and 2398 for the hospital surgical suite.

3. The case from Step 2 was scheduled into one of the two ORs from Step 1. Scheduling was done at random by using Earliest Start Time or Latest Start Time. Scheduling at random was done by generating a uniformly distributed random number and scheduling the case into OR 1 if it was $<0.5$ and into OR 2 otherwise. For the latter two heuristics, estimated start times were obtained from the means of the series' cases' procedures' historical durations. These would be the surgical suite's scheduled start times.

4. The actual overutilized hours achieved by scheduling the case into the chosen OR was calculated by using the retrospective knowledge of how 
long each case took. This was taken as the difference between the duration of the regularly scheduled workday and the actual total hours of cases and turnover times from Steps 1 and 2.

5. The minimum possible overutilized hours was obtained from the series' and cases' actual durations.

6. The first through fifth steps were repeated 99 times.

7. The sums of the 100 overutilized hours from the fourth and fifth steps were calculated. The difference was taken between the two sums and then divided by 100 .

8. The first through seventh steps were repeated 99 times.

9. The mean and SE of the resulting 100 differences from Step 8 were calculated.

To calculate the overutilized hours in the fourth and fifth steps, we needed to specify a duration for the regularly scheduled workday. The choices are described in the last paragraph of Methods. For each choice of the duration of the regularly scheduled workday, there were 20,000 simulated ORs, 10,000 with the case put into it and 10,000 without. We calculated the percentage of ORs that had overutilized hours by using all 20,000 simulated ORs and the SE of the percentage.

\section{References}

1. Strum DP, Vargas LG, May JH, Bashein G. Surgical suite utilization and capacity planning: a minimal cost analysis model. J Med Syst 1997;21:309-22.

2. Dexter F, Epstein RH, Marsh HM. Statistical analysis of weekday operating room anesthesia group staffing at nine independently managed surgical suites. Anesth Analg 2001;92:1493-8.

3. Strum DP, Vargas LG, May JH. Surgical subspecialty block utilization and capacity planning: a minimal cost analysis model. Anesthesiology 1999;90:1176-85.

4. Dexter F, Traub RD. Determining staffing requirements for a second shift of anesthetists by graphical analysis of data from operating room information systems. AANA J 2000;68:31-6.

5. Ozkarahan I. Allocation of surgical procedures to operating rooms. J Med Syst 1995;19:333-52.
6. Dexter F, Traub RD. Statistical method for predicting when patients should be ready on the day of surgery. Anesthesiology 2000:93:1107-14

7. Dexter F, Macario A, Traub RD. Which algorithm for scheduling add-on elective cases maximizes operating room utilization? Use of bin packing algorithms and fuzzy constraints in operating room management. Anesthesiology 1999;91:1491-500.

8. Epstein RH, Dexter F. Statistical power analysis to estimate how many months of data are required to identify operating room staffing solutions to reduce labor costs and increase productivity. Anesth Analg 2002;94:640-3.

9. Dexter F. A strategy to decide whether to move the last case of the day in an operating room to another empty operating room to decrease overtime labor costs. Anesth Analg 2000;91:925-8.

10. Dexter F, Traub RD, Qian F. Comparison of statistical methods to predict the time to complete a series of surgical cases. J Clin Monit Comput 1999;15:45-51.

11. Dexter F, Traub RD, Lebowitz P. Scheduling a delay between different surgeons' cases in the same operating room on the same day using upper prediction bounds for case durations. Anesth Analg 2001;92:943-6.

12. Solovy A. Benchmarking guide 99. Hosp Health Netw 1999;73: 49-62.

13. Zhou J, Dexter F. Method to assist in the scheduling of add-on surgical cases: upper prediction bounds for surgical case durations based on the log normal distribution. Anesthesiology 1998; $89: 1228-32$.

15. Strum DP, May JH, Vargas LG. Modeling the uncertainty of surgical procedure times: comparison of the log-normal and normal models. Anesthesiology 2000;92:1160-7.

14. Birge JR, Maddox MJ. Bounds on expected project tardiness. Oper Res 1995;43:838-50.

16. Dexter F, Macario A, Traub RD, et al. An operating room scheduling strategy to maximize the use of operating room block time: computer simulation of patient scheduling and survey of patients' preferences for surgical waiting time. Anesth Analg 1999;89:7-20.

17. Strum DP, Sampson AR, May JH, Vargas LG. Surgeon and type of anesthesia predict variability in surgical procedure times. Anesthesiology 2000;92:1454-66.

18. Macario A, Dexter F. Estimating the duration of a case when the surgeon has not recently performed the procedure at the surgical suite. Anesth Analg 1999;89:1241-5.

19. Dexter F, Macario A, O'Neill L. A strategy for deciding operating room assignments for second-shift anesthetists. Anesth Analg 1999;89:920-4.

20. Dexter F, Macario A, Lubarsky DA, Burns DD. Statistical method to evaluate management strategies to decrease variability in operating room utilization: application of linear statistical modeling and Monte-Carlo simulation to operating room management. Anesthesiology 1999;91:262-74.

21. Wright IH, Kooperberg C, Bonar BA, Bashein G. Statistical modeling to predict elective surgery time. Anesthesiology 1996; $85: 1235-45$ 